\section{The Politics of Fear: Médecins Sans Frontières and the West African Ebola Epidemic}

\author{
Michiel Hofman and Sokhieng Au, editors; Oxford \\ University Press, New York, New York, USA; ISBN-13: \\ 978-0190624477; ISBN-10: 0190624477; Pages: 304; \\ Price: US \$15.72
}

$\mathrm{T}$ he Politics of Fear: Médecins Sans Frontières and the West African Ebola Epidemic is an engaging collection of essays that critically evaluates the response of Médecins Sans Frontières (MSF) to the Ebola epidemic in West Africa during 2014 and 2015. It is an appropriate mixture of reflections, vignettes, case studies, and critiques regarding the role of MSF in the epidemic. It does not offer detailed background

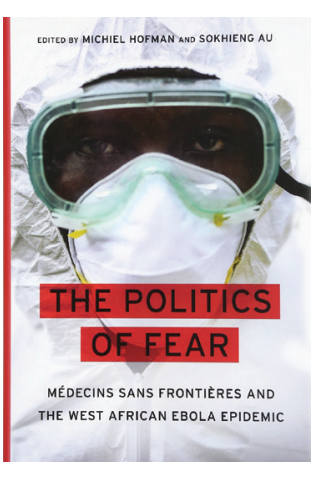
on the organization itself or on the Ebola epidemic. Thus, the target audience includes readers who understand the role of MSF, as well as the basic principles of Ebola virus disease (EVD). The book describes complexities of management of such a large outbreak and provides context for the difficult decisions made by MSF leadership, as well as clinicians on the ground. Many books and articles have been written about the epidemiology of EVD, but this book is unique in its focus on the politics and ethics of caring for EVD patients in a complex sociopolitical environment.

The content is well balanced, and chapter authors include MSF physicians, MSF nurses, health officers, administrators, social anthropologists, and bioethicists. MSF as an organization is presented as neither savior nor villain in the crisis; it is described objectively as a player whose actions were motivated by the epidemic itself and its decisions as difficult choices at a time where there were no right choices. The essays eloquently describe the motivations behind such decisions as quarantine of patients, participation in clinical trials, allocation of resources, evacuation of medical personnel, and use of experimental treatments. Each account provides a unique perspective of different aspects of the epidemic and response. In these descriptions, the authors highlight the complicated dynamics in navigating limited resources, overwhelming disease, and citizen distrust. Permeating these essays is the humanity and courage of the doctors, nurses, and staff caring for patients.

This book achieves its goal of describing the multifaceted considerations that must be taken into account when designing policy and response to an epidemic and highlighting the vulnerabilities of healthcare systems that must be addressed to achieve more effective response. It also raises the argument that global humanitarianism, although motivated by altruism, must adapt to existing economies, cultures, and politics if there is any hope for a successful outcome. Although the description of the MSF response is powerful, the essay structure of the multiauthored book does not necessarily provide a coherent approach or strategy to avoid and more effectively treat future crises. However, the book does an outstanding job of outlining the problems that must be addressed.

This book is not a detailed description of the science or epidemiology of EVD but provides in-depth explanation and assessment of the actions of MSF in response to the Ebola epidemic. It is an appropriate resource for those readers who wish to better understand the intricacies of a global epidemic humanitarian response in the context of the disparate sociopolitical environments in which they exist. The reader will appreciate the courage of MSF healthcare providers in the response to the Ebola epidemic, as well as the problems that must be addressed to ensure the dedication and sacrifice of these providers translate to favorable outcomes.

\section{Keith Hamilton}

Author affiliation: University of Pennsylvania, Philadelphia, Pennsylvania, USA

DOI: https://doi.org/10.3201/eid2311.171206

Address for correspondence: Keith Hamilton, Division of Infectious Diseases, Hospital of the University of Pennsylvania, Ste 110, Silverstein Bldg, 3400 Spruce St, Philadelphia, PA 19104, USA; email: keith. hamilton@uphs.upenn.edu

\section{Ebola: Profile of a Killer Virus}

Dorothy H. Crawford; Oxford University Press, New York, NY, USA, 2017; ISBN-10: 0198759991; ISBN-13: 978-0-19-875999-7 Pages: 205; Price: US \$27.95

Qince its first identification in $\checkmark 1976$, Ebola has been responsible for about 2 dozen lethal outbreaks in Africa and numerous horrific Hollywood films. Then, in 2014, life seemed to imitate art when Ebola rapidly tore through Guinea, Sierra Leone, and Liberia, causing an unprecedented 11,300 deaths. In this very readable book, Dorothy Crawford, a microbiolo-

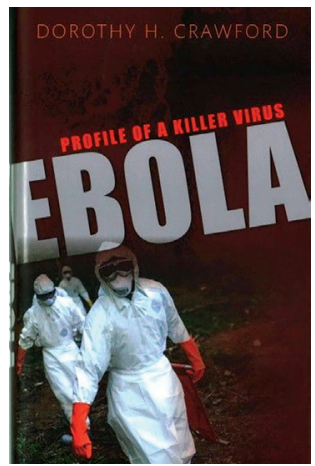


gist and the author of several previous books on infectious diseases for the general reader, helps demystify Ebola. The book gives a concise overview of Ebola, beginning with the first identified outbreaks in 1976, and moves on to a summary of what we know about the virus and the disease, clinical care, and attempts to identify the natural reservoir. Another chapter deals briefly with outbreaks after 1976, before moving swiftly to the biggest surprise, Ebola's eruption in West Africa in 2014.

The book is not intended for the technical expert but is an accessible capsule summary for the general reader. Given its origin, the book is also understandably focused on events and perspectives in the United Kingdom. The descriptions in the book are clear and often vivid, and the book provides an accessible overview of Ebola to date. The story has its share of heroes, of course, and is eventful enough for a Hollywood thriller. There are hardworking (and frightened) Western professionals and dedicated nuns. It is especially gratifying to see some of the African heroes recognized, such as JeanJacques Muyembe, a microbiology professor in Zaire and the first trained professional to witness the Ebola cases in 1976, who lived to use his knowledge in many later outbreaks. The book is enlivened by memorable vignettes. During the 1995 outbreak in Kikwit (Zaire), Dr. Muyembe explains Ebola and the appropriate precautions to local villagers, translated into their own cultural context as evil spirits that could escape to anyone who touches the victim. Another section tells how a group of young ruffians in a Liberia slum, Moa Wharf, became leaders in the local fight against Ebola.

One of the most disquieting things about the Ebola outbreak in West Africa was the sluggish response of the international community, the subject of much soulsearching ever since. The major response was announced in mid-September and began in October 2014-more than 6 months after the first public report of the outbreak in March. Ironically, newly built Ebola treatment units remained unused as patient numbers dwindled, and by the time vaccine and drug candidates became available, there were no longer enough patients for full-scale randomized trials.

The official goal of "getting to zero" (no Ebola cases) seems implausible, not just because of chronic transmission but because the virus is part of the local ecology. A growing body of circumstantial evidence, including a paper published in Emerging Infectious Diseases a few years ago (1), suggests that the virus has been in West Africa for some time. The discoveries of Bundibugyo virus in Uganda, unknown until 2007, and the Taï Forest Ebolavirus species in Côte d'Ivoire should have debunked the notion that Ebola could not be present in West Africa.
Crawford does an excellent job of describing the medical response and the urbanization of the outbreak, which probably accounted for much of its devastation and may be a portent of things to come. The usual narrative of the response is generally that "we" came in force and vanquished the outbreak. Because it is obvious and compelling, medical treatment tends to get the most attention, with public health and community participation often relegated to smaller supporting roles. Although Crawford discusses community mobilization, especially late in the epidemic, readers might be interested to know more about this part of the story. Anthropologists and some organizations have been working on documenting and improving grassroots efforts by the people of Africa, including community health workers. As we strengthen health systems and improve access to primary care, we also need to put in place basic public health measures (including water, sanitation, hygiene, and surveillance) that can make communities more resistant to infection, and develop relationships with local leaders before outbreaks occur.

Sustaining the gains achieved has been the hardest part, but is crucial. The author describes a new Ebola Transition Group and Institute for Sanitation, Water, and Public Health in Sierra Leone that appear to be hopeful starts. These measures can help with the next Ebola outbreak but also with the inevitable unexpected epidemic. The author, noting that the Zika virus outbreak in the Western Hemisphere followed shortly after the Ebola outbreak (and was similarly unexpected), rightly argues for more comprehensive global capability. Other infections are waiting for an opportunity to emerge, and the current reactive strategy will likely repeat the same mistakes. George Bernard Shaw wrote: "We learn from history that men [sic] never learn anything from history." As Crawford's book shows, it's time we learned.

\section{Reference}

1. Schoepp RJ, Rossi CA, Khan SH, Goba A, Fair JN. Undiagnosed acute viral febrile illnesses, Sierra Leone. Emerg Infect Dis. 2014;20:1176-82. http://dx.doi.org/10.3201/eid2007.131265

\section{Stephen S. Morse}

Author affiliation: Columbia University/Mailman School of Public Health, New York, New York, USA

DOI: http://dx.doi.org/10.3201/eid2211.171207

Address for correspondence: Stephen S. Morse, Columbia University, Mailman School of Public Health, 722 W 16th St, \#1504, New York, NY, 10032, USA; email: ssm20@columbia.edu 